Review Article

\title{
Futuristic Cyber-Twin Architecture for 6G Technology to Support Internet of Everything
}

\author{
Sapna Juneja $\mathbb{D},{ }^{1}$ Mamta Gahlan $\left(\mathbb{D},{ }^{2}\right.$ Gaurav Dhiman $\mathbb{D D}^{3}$ and Sandeep Kautish $\mathbb{D}^{4}$ \\ ${ }^{1}$ IMS Engineering College, Ghaziabad, Uttar Pradesh, India \\ ${ }^{2}$ Maharaja Surajmal Institute of Technology, Delhi, India \\ ${ }^{3}$ Department of Computer Science, Government Bikram College of Commerce, Patiala 147001, Punjab, India \\ ${ }^{4}$ LBEF Campus, Kathmandu, Nepal \\ Correspondence should be addressed to Sandeep Kautish; dr.skautish@gmail.com
}

Received 4 September 2021; Accepted 27 September 2021; Published 6 October 2021

Academic Editor: Yi-Zhang Jiang

Copyright (C) 2021 Sapna Juneja et al. This is an open access article distributed under the Creative Commons Attribution License, which permits unrestricted use, distribution, and reproduction in any medium, provided the original work is properly cited.

\begin{abstract}
With the rapid growth of Internet of Everything, there is a huge rise in the transportable Internet traffic due to which its associated resources have exceptional obstacles which include reliability, security, expandability, security, and portability which the current available network architectures are unable to deal with. In this paper, an IoT centric cyber-physical twin architecture has been proposed for 6G Technology. The cyber-twin technology helps out in serving stronger communication and also contains several features that help out in assisting communication like maintaining a log record of network data and managing all digital assets like images, audio, video, and so forth. These features of the cyber-twin technology enable the proposed network to deal with those exceptional obstacles and make the system more reliable, safe, workable, and adaptable.
\end{abstract}

\section{Introduction}

The population of the world is growing like a bomb and the people are now connected with each other with the help of millions of devices. The available networks will be insufficient to accomplish the rising demand of billions of devices thereafter. Thus, the presumed future network should be able to gratify the intense network traffic and its associated services. In the current times, Internet of Everything seems to be a future network system that can attain rational connections between humans and devices with the help of various technologies like Machine Learning and 5G. An Internet of Everything enabled network architecture to reinforce the complex features of data like collecting pervasive data, data clustering, refining, and lastly allocating it for utilization.

\section{Literature Review}

The current network architecture suffers from the scalability problem, that is, not being able to fulfill the tremendously increasing demand of Internet services and devices. Further, in order to secure the network, all the accessed devices can be kept under observation to detect any unauthorized access but it is not feasible to keep a watch on every user accessing the network. Thus, these problems lead to the security breach in the network. Another major problem faced by current available networks is that maintaining collaboration and synchronization among various resources controlled by multiple network service providers is very burdensome which finally affects the quality of the service of the network. All these problems faced by available network architectures are unfavorable and thus hinder the growth and development of the services provided by the available networks. For dealing with such issues and challenges, many researchers tried to propose various kinds of network architectures, each with its own pros and cons. Few of them are named as Named Data Networking, DONA, Content Centric Networking, and so forth but still these networks are not selfsufficient to support huge traffic as these networks are incompatible with current IP networks [1].

One of the architectures named MobilityFirst [2] was developed to deal with current issue related to network but it 
was lacking the security of the whole network. Another architecture proposed was Expressive Internet Architecture [3] which was better than the former in terms of network security as it was incorporating various protocols used to control and manage the network but it also has some pitfalls associated with it like difficulty in network expandability and relatively high cost of establishing and managing the network. ChoiceNet [4] was developed to facilitate choices and the financial relationships among the various entities of the network but it is not much efficient for complex real-time services. Further in the queue, a cloud-based architecture had also emerged with the potential of sharing network resources wisely and managing the immense data gathered from IoT or IoE devices such as CloNe, Cloud Integrated Networks [4], and Nebula. Nevertheless, these networks also face performance issues due to the low computational ability of the connected devices. Another problem associated with these networks is the lack of mobility as it is incapable of serving the mobile devices, although the mobile devices are much trending nowadays. One of the types of cloud-based network architectures is CloNe architecture. It was beneficial for cloud customers as it helps to connect network and data domains. Apart from that, network resources are spread properly in the whole network among various devices, thus providing better connectivity to the end user. These networks are unable to handle mobility in the network though. The Cloud Integrated Network architecture [5] fixes the cloud of the network edge to the network itself, thus providing the optimal throughput. It is efficient in the terms that it manages the resource distribution and utilization credibly but it ist not able to deal with mobility issue of the devices in the cloud. Hence, a variety of network architectures have been defined by the researchers but still they are not appropriate for implementing as they encounter multiple challenges like mobility, expandability, security and so forth. In this research paper, cloud-based cyber-twin architecture [6] has been proposed as the futuristic network. By using cyber-twin architecture, we can implement a novel architecture of network that must be able to deal with the issues of mobility, expandability, security, and reliability. Table 1 represents the work done by various researchers in the field of cyber-twin technology:

\section{The Concept of Cyber-Twin}

Because of the sudden rises in the digitalization of the whole world in Industry 4.0, the concept of cyber-twin arose. The main motive behind the development of cyber-twin is to support Industry 4.0 in such a manner that the production environment becomes more flexible, productive, and resource-efficient. In order to make the production more fast and progressive, the most important constraint is the minimization of the response time to the customer's query and this can be fulfilled only when there exists a network that is fast enough to manage everything within a blink [15]. This fast network can be supported using cyber-twin. Before moving to the concept of cyber-twin, some basic terminologies need to be studied for understanding the purpose of cyber-twin.
3.1. Cyber-Physical System. Cyber-physical systems are the systems that act as a base for Industry 4.0. The structure of the cyber-physical system is a collection of hardware and software that work together with embedded system. Embedded systems [16] are being used to manage the sensors and their states present in the cyber-physical systems. Cyberphysical systems can be summed up as combination of hardware and software, where the software affects the behavior of the system. Further, the cyber-physical system can visualize the physical components in real-time environment and hence can be used for simulation of the physical components. Any function is being performed by the cyberphysical system by accessing the recorded data.

3.2. Digital Twin Paradigm. A digital twin is a virtualization of any physical system that keeps on updating every time by analyzing the performance of the previous one. If the original physical system and its twin copy are integrated with each other and share the data and information, the twin is known as digital twin [17]. So, in such scenario, if the changes are being done in one system, these changes will automatically reflect to the other system and vice versa. Ontology based digital twins can be used to implement and manage the digital twins in current data ecosystem. Figure 1 shows the structure of digital twin.

3.3. Cyber-Physical Twin. Cyber-physical twin system is a key methodology that entitles the products which are viable and reliable in terms of their services. The system is composed of two-level hierarchies, cyber-twin and physical twin. Both twins associate with each other for completing the life cycle of cyber-physical system. The physical twin is made up of actual modules, that is, sensors and actuators. Sensors are being used by the physical twin to instigate the impact of environment [18]. The data thus collected from the sensors has been sent to the cyber-twin which further analyzes the data and, depending on this analysis, provides feedback to the physical twin and the user. Communication between the user and the twins can be achieved with the help of an interface. The interface is also used for controlling the behavior of the physical twin and analyzing the reaction of the cyber-twin. The next section contains the behavior of cybertwin and physical twin and also discusses the mode of interaction between them [19].

3.3.1. Cyber-Twin. The cyber-twin is pragmatic approach of any physical component of the cyber-physical twin that incorporates the response of the physical component. Hence, the cyber-twin behaves much similar to the physical component. The core of the cyber-twin embraces a model that contains all the relevant information and its associated relationships. The data in this model has been stored by the available sensors of the system and can be used later on for performing computations. Thus, this model of cyber-twin contains a database that accommodates the data used for carrying out the operations by physical twin [20]. The cyber-twin generates an algorithm which transforms the behavior of the physical components. 
Further, by applying the simulation technique, the present situation and the upcoming situation and the functions of the physical twin can be predicted.

3.3.2. Physical Twin. A physical twin is a term similar to digital twin, which elaborates the physical systems or products of the real world, which further is replicated by digital twin. The physical twin is enabled with the sensors that are provided to keep track of the functions and the behavior of the components of the product during the usage phase of the product. Further, these physical components work in real-time environment with the help of actuators. An interface is also added to the physical twin to perceive the interactivity between the physical twin and cyber-twin.

\subsubsection{Communication between Physical Twin and Cyber-} Twin. The communication between the physical twin and cyber-twin must be made in such a way that there should be no break in the communication between them. Thus, realtime communication should be incorporated among the twins. In order to pursue this real-time communication between the twins, various protocols like MQTT and so forth can be implemented. The steps followed for the communication between the physical twin, cyber-twin, and the end user can be elaborated as follows:

(1) The requirements must be gathered as input that must meet the choice of individual user. These requirements further help out in the configuration of cyber-physical twin system.

(2) The next step is to configure the cyber-physical twins precisely and carefully. This configuration is a twostep process in which the first step is the implementation of the sensors, which further forms the physical part of the cyber-physical twin. In the second step, the virtual twin of the respective cybertwin is created.

(3) Both processes are connected together and reconfigure the database of the cyber-twin. This reconfiguration of the database is aligned by the sensors and the simulation models.

(4) Further, sensors are being used to quantify the real physical influences of the environment.

\section{Structure of Cyber-Twin Network}

The proposed futuristic structure of cyber-twin network for 6G is composed of four parts: parent cloud, child cloud, user interface, and cyber-twin. Figure 2 shows the proposed structure of cyber-twin network.

The functions of each of the part of cyber-twin architecture are as follows:

(1) Parent cloud: Various clouds are connected to each other to form the parent cloud. This parent cloud establishes the base of the network. Connection between these clouds can be of any type like via satellite or fiber optics. The difference between the existing cloud and future cloud would be that they would not only be used for storing and computation of data; instead these parent clouds will also be used for calculation, caching, and sharing resources efficiently among various end users.

(2) Child cloud: This structure is a three-level hierarchy where the topmost level is for parent cloud and the bottom level is for user interface. The child cloud lies in between the parent cloud and child cloud. Thus, child cloud is used to serve the functionality to the user through user interface as provided by parent cloud. Other than this, the parent cloud provides more features to the user in comparison to the child cloud. It can easily process out the user's request as it is adjacent to the user interface.

(3) User interface: User interface here represents the interface that has been used to provide the connectivity between the clouds and users and further user refers to various devices connected to the network being used by the individual user. So, if any user wants to access the data or share the resources with any other user in the network, a request is made by user, which will be further sent to the child cloud and then to parent cloud. The user needed to be connected to the cloud all the times through cybertwin for performing any computation, instead of being connected to the server.

(4) Cyber-twin-It is the most important part of the futuristic network. It has been positioned adjacent to the child cloud and the communication it provides is different from the communication provided by the existing networks; thus it is capable of supporting many services which have not been provided by the present communication models [21].

\section{Precedence of Cyber-Twin System over Peer- to-Peer Communication System}

Futuristic cyber-twin network architecture supercedes the present peer-to-peer architecture in so many ways and the most important functionalities provided by the cyber-twin which the peer-to-peer system is unable to yield are the following:

(a) Cyber-twin has the ability to keep a log record of the data of all the users connected via cyber-twin network which remains shielded as no external user is allowed to access the log record of cyber-twin, while, in the case of peer-to-peer architecture, various ISPs keep track of all the log records of the end user which further can be easily hacked by a hacker [15].

(b) Cyber-twin is a way of representing any entity digitally. This entity can be either human being or any object. In the physical world, cyber-twin has been used to assist the entity. While assisting the user, the cyber-twin architecture requires the end user to get connected to the cyber-twin which further communicates with the cloud (child and parent) for 
Table 1: A critical review of significant similar contributions from researchers.

\begin{tabular}{|c|c|c|c|}
\hline $\begin{array}{l}\text { S. } \\
\text { no. }\end{array}$ & $\begin{array}{l}\text { Author } \\
\text { and year }\end{array}$ & Methodology & Findings \\
\hline 1. & [7] & Cloud operated cyber-twin architecture has been defined. & $\begin{array}{l}\text { Architecture is very efficient for high speed networks but } \\
\text { lacks privacy and security of the data. }\end{array}$ \\
\hline 2. & {$[8]$} & $\begin{array}{l}\text { Digital twin architecture has been defined by taking the } \\
\text { concept of data analytics and virtual reality. }\end{array}$ & $\begin{array}{l}\text { Future industrial applications of digital twin architecture } \\
\text { are explained. Various objectives that can be used for } \\
\text { improvisation of digital twin technology are elaborated. }\end{array}$ \\
\hline 3. & [9] & $\begin{array}{l}\text { A five-layered architecture of cyber-physical system is } \\
\text { proposed which can further be implemented in Industry } \\
4.0 \text { for efficient production management. }\end{array}$ & $\begin{array}{l}\text { The paper provides practical guidelines to manufacturing } \\
\text { organizations for the implementation of cyber-physical } \\
\text { systems for intelligent manufacturing process. }\end{array}$ \\
\hline 4. & {$[10]$} & $\begin{array}{l}\text { Cyber-physical twins and their associated concepts are } \\
\text { reviewed in the paper. }\end{array}$ & $\begin{array}{l}\text { Advantages of cyber-physical twins are evaluated. The } \\
\text { behavior and the usage of cyber-twins in real time } \\
\text { environment are stated in the paper. }\end{array}$ \\
\hline 5 & {$[11]$} & $\begin{array}{c}\text { In this paper, a four-layer reference model for digital twin } \\
\text { has been proposed. This reference model contains } \\
\text { guidelines for risk prevention and prediction using digital } \\
\text { twin for enhancing the safety of employees at their } \\
\text { workplace. }\end{array}$ & $\begin{array}{l}\text { The proposed digital twin model is very useful for the } \\
\text { organizations where employees live in high risk } \\
\text { manufacturing environment as the proposed model is } \\
\text { capable of developing virtual modeling environment for } \\
\text { the manufacturing process, thus identifying the risks } \\
\text { involved. }\end{array}$ \\
\hline 6. & {$[12]$} & $\begin{array}{l}\text { Integration of discrete event simulation and digital twin } \\
\text { has been explained in the paper and the challenges of } \\
\text { contemporary logistics have been picked out. }\end{array}$ & $\begin{array}{l}\text { Digital twins can be used for enhancing the functionality of } \\
\text { DES for real-time simulation environment. }\end{array}$ \\
\hline 7. & {$[13]$} & $\begin{array}{l}\text { An approach for designing digital twin technology for } \\
\text { communication system has been used. Implementation of } \\
\text { the model is done on Amazon Web Services. }\end{array}$ & $\begin{array}{l}\text { The proposed system has the ability to act as the replica of } \\
\text { the future power system. }\end{array}$ \\
\hline 8. & {$[14]$} & $\begin{array}{l}\text { A digital driven framework for designing of products has } \\
\text { been represented and a case study has been used to show } \\
\text { the advantages of this proposed framework. }\end{array}$ & $\begin{array}{l}\text { The proposed approach seems useful for redesigning of an } \\
\text { existing product instead of designing the whole system } \\
\text { from the beginning. }\end{array}$ \\
\hline
\end{tabular}

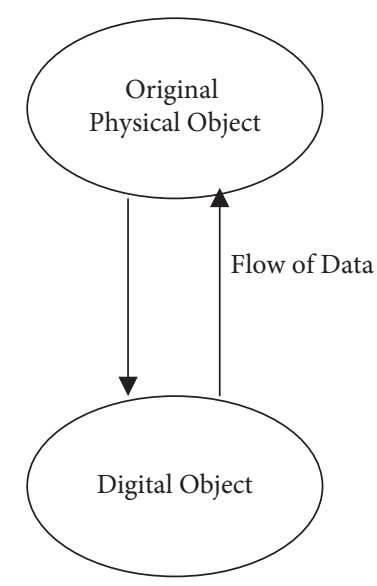

FIGURE 1: Structure of digital twin.

accomplishing the request done by the end user, while in the peer-to-peer network the end user directly connects to the cloud for obtaining the services [8]. Thus, in the former case, the user achieves better quality of service due to the cyber-twin's ability of knowing the user requirements well.

\section{Advantages of Cyber-Twin System}

If required resources of the cyber-twin networks will be available in proximity and if used efficiently, cyber-twin networks can have a revolutionary impact in the digital world.
The following section presents the benefits offered by the cyber-physical twin network to the entire community:

(1) As the cyber-twin system gathers a lot of data for all the communication over the entire network, this collected data can further be utilized to enhance the properties of next system which is to be developed for the ease of use by the user and thus helps in developing a more better system that must be able to fulfill the user's requirements in all aspects [22].

(2) In cyber-twin system, the lifecycle maintains a parallel two-phase process in which the first phase involves execution of the current cyber-twin system 


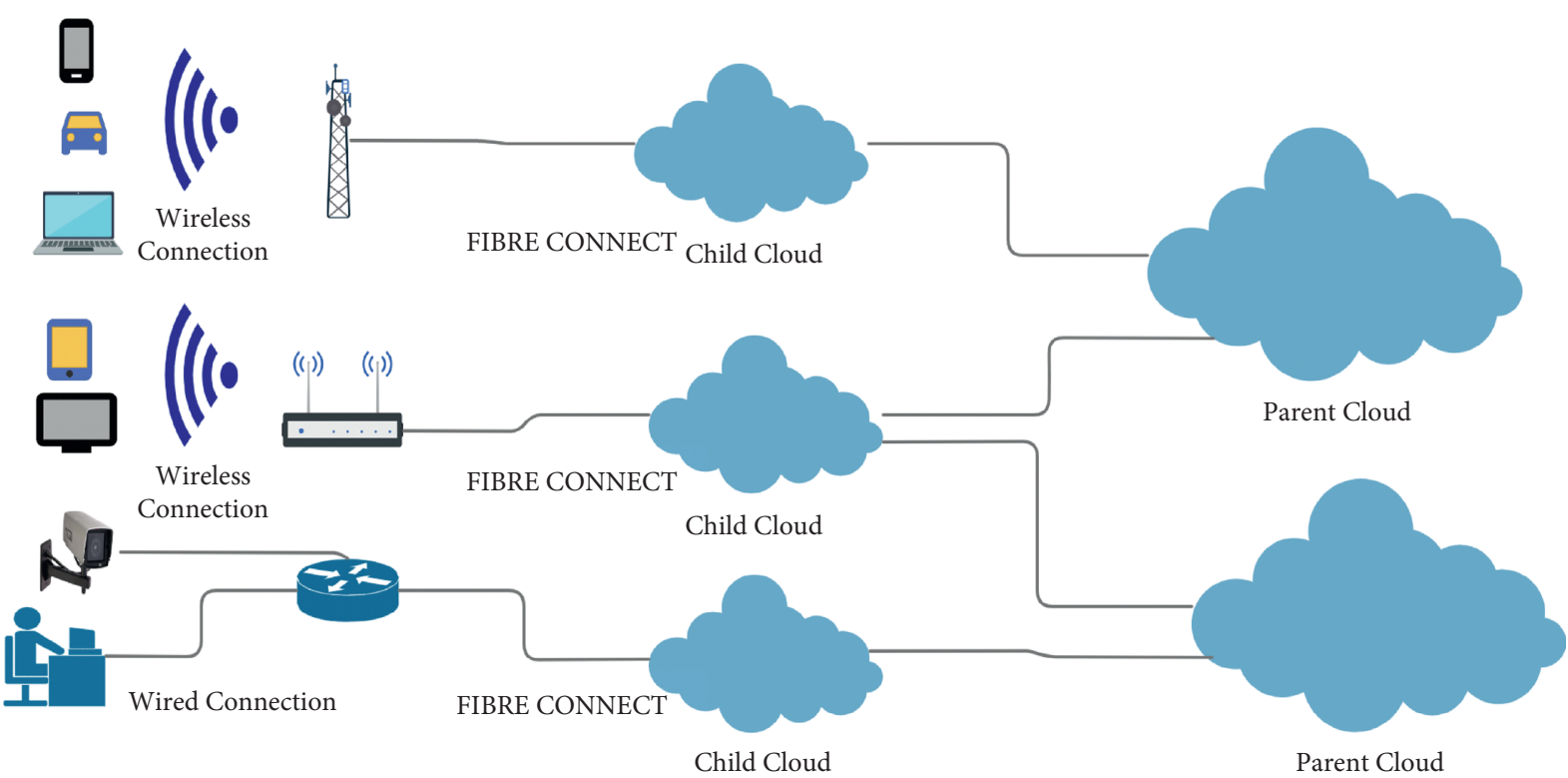

FIgURE 2: Structure of cyber-twin network.

and the second phase involves modifications in the existing system depending upon the current behavior of the system. Hence, two processes keep on continuing all the times which does not affect the throughput of the system.

(3) In any of the traditional networks, all the hardware involved has some lifetime and once the lifetime gets over, the hardware should be replaced by the new component, which ultimately affects the performance of the system as, during the replacement process of the component, the whole communication process gets stopped. Meanwhile, in the case of cyber-twin system, the lifetime of any of the components of the whole network can be easily predicted and thus it can be replaced before reaching the end. As the cyber-twin system leads to more accuracy and better performance and thus prevents the system from getting failed due to component failure, it has more reliability in comparison to traditional networks.

(4) As the number of mobile devices in the network is growing very fast, some mechanism is needed to take care of mobile data as the conventional network architecture is insufficient to serve mobile data because the present networks manage the data traffic by taking host address into account, which remains stationery. In the cyber-twin network architecture, every entity, either human or device, is represented by a digital ID not by host address which is mobile and thus cyber-twin network allows mobility of traffic for the convenience of users [23].

(5) The cyber-twin network architecture provides more security to the data as it has several authentication processes running in the background which first confirms whether the user or the device is authentic and only then it allows the entity to access the data of the cyber-twin network. Different security mechanisms are imposed on different entities like if a human being wants to access the data; the retina of the eye is scanned first to give access to the user which is somehow better than the security mechanism provided by current network architectures as these architectures do not contain such tough constraints on the access of the data.

\section{Cyber-Twin Applications in Real- World Environment}

This section of the review focuses on the application areas of cyber-twin in real-world environment ranging from healthcare and manufacturing to development of smart cities.

(1) Healthcare: The most important application area of cyber-twin is the healthcare sector. As the healthcare sector is now growing exponentially due to the usage of IoT devices, the implementation of cyber-twin makes it possible to share the large amount of data collected from IoT devices at a very high speed which the traditional networks are not able to do with the same accuracy and speed. Secondly, cyber-twin can also be used for simulating the drugs' effects on the human body as well as planning and execution of medical therapeutics. Cyber-twin is also beneficial in AI-enabled healthcare for decision- and predictionmaking especially in the situations where it seems difficult for the physicians to take the decision about any medical treatment for any patient. Digital twin has the ability to make the decisions based on reallife situations [24].

(2) Manufacturing: Nowadays, manufacturing industry is looking for the system that can provide high connectivity and should be able to keep a track of the 
products. These applications to manufacturing industry are being supported by cyber-twin. Digital twin has the ability to track the performance of the machines in manufacturing industry in real-time environment and also to predict the lifetime and future performance of the machine which is very important for performance and reliability measuring for the product development. Products can be easily tested by setting up a simulation environment by the cyber-twin [21].

(3) Development of smart cities: The smart cities are now growing very fast because of the increase in IoT devices; people are coming more closer to each other for sharing information and, in this scenario, digital twins are required to connect all these communities together. The cyber-twin can help out in planning of smart cities by using AI techniques and can also be used to model smart buildings, smart traffic management, smart farming and smart livestock, and so forth [25].

\section{Challenges of Cyber-Twin Technology}

It is now apparent that cyber-twin requires various technologies to run in parallel with it and thus faces a lot of challenges. So, in order to deal with these challenges, the first task is to identify them. In this section, some challenges faced by digital twin technology will be discussed so that they must be taken into account in the development of the cyber-twin system.

(1) The cyber-twin architecture requires infrastructure that must be compatible with the various upcoming technologies like AI and IoT that will run in collaboration with the cyber-twin for the effective running of the entire network. Without a compatible and well-defined infrastructure, the cyber-twin will fail in achieving its goal, resulting in a malfunction in the system.

(2) The second challenge that appears is the data required for cyber-twin. The required data should be of high quality without any interruption in between. Interrupted and low-quality data leads to the degradation in performance of cyber-twin. Some analysis of the collected data should be there to check whether the data is perfect in all aspects and does not carry any uncertainty in it for the smooth functioning of cyber-twin architecture [26].

(3) Another major challenge concerned with the cybertwin architecture is the privacy of the sensitive data which it obtains from the multiple IoT devices connected to it. So, for dealing with the data security related to cyber-twin, some authentication and security mechanism should be applied at the IoT device to prevent it from unauthorized access, thus protecting the cyber-twin data [27].

(4) Despite all these challenges, the next challenge that comes into light is that no standard design has been available for the development of cyber-twin and if the system will be developed lacking standard design, there will be no uniformity throughout which will mislead the information flow, thus resulting in the imperfect system not capable of achieving its goal (A. [28]).

(5) The last challenge faced by digital twin technology is that digital twin requires more maintenance and people are neither well equipped nor skilled in the maintenance of cyber-twin because of lack of availability of resources required for cyber-twin.

\section{Conclusion and Future Work}

The paper deals with the definition and the concepts of cyber-twin and the representation of structure of futuristic cyber-twin network. Further, the comparison between the cyber-twin network and peer-to-peer network has been elaborated to show how cyber-twin networks are better than peer-to-peer networks in all aspects. Advantages and applications of cyber-twin in real-world environment are discussed along with the challenges the cyber-twin will face if adopted for 6G Technology. Cyber-twins are a novel and revolutionary approach in the field of Industry 4.0 which enables the escalation in the reliability or sustainability of any component.

Some topics that still need to be explored to achieve more secure, innovative, and expandable architecture are as follows:

(1) How the cyber-twin networks can be intergraded into new business models fruitfully must be explored. The aim of usage must be defined.

(2) Well-planned authentication framework for the cyber-twin must be developed to trace the behavior of the network.

(3) For obtaining more efficiency and quality of services, artificial intelligence can be used for implementation and association among various cyber-twins.

(4) Resource management is a big issue while using the clouds in the cyber-twin network which can be managed by Blockchain technology for efficient allocation of resources among various devices.

\section{Data Availability}

The data used to support the findings of this study are available upon request via e-mail: gdhiman0001@gmail.com.

\section{Conflicts of Interest}

The authors declare that they have no conflicts of interest.

\section{References}

[1] A. M. Baharudin and W. Yan, "Long-range wireless sensor networks for geo-location tracking: design and evaluation," in Proceedings of the 2016 International Electronics Symposium, IES 2016, pp. 76-80, Denpasar, Indonesia, September 2017. 
[2] S. Li, Y. Zhang, D. Raychaudhuri, and R. Ravindran, "A comparative study of MobilityFirst and NDN based ICN-IoT architectures," in Proceedings of the 2014 10th International Conference on Heterogeneous Networking for Quality, Reliability, Security and Robustness, QSHINE 2014, vol. 1, pp. 158-163, Rhodes, Greece, August 2014.

[3] Z. Meng, Z. Chen, and Z. Guan, "Peer-to-peer file sharing in next generation eXpressive Internet Architecture," CCF Transactions on Networking, vol. 1, no. 1-4, pp. 28-36, 2019.

[4] B. Gaujal, S. Haar, and J. Mairesse, "Blocking a transition in a free choice net and what it tells about its throughput," Journal of Computer and System Sciences, vol. 66, no. 3, pp. 515-548, 2003.

[5] A. Albugmi, M. O. Alassafi, R. Walters, and G. Wills, "Data security in cloud computing," in Proceedings of the 5th International Conference on Future Generation Communication Technologies, FGCT 2016, pp. 55-59, London, United Kingdom, August 2016.

[6] E. Pignaton, D. F. Joanna, I. Olszewska et al., "Ontological concepts for information sharing in cloud robotics," Journal of Ambient Intelligence and Humanized Computing, Article ID 0123456789, 2020.

[7] Q. Yu, J. Ren, H. Zhou, and W. Zhang, "A cybertwin based network architecture for 6G," in Proceedings of the 2nd 6G Wireless Summit 2020: Gain Edge for the 6G Era, 6 G SUMMIT 2020, Levi, Finland, March 2020.

[8] K. Y. H. Lim, P. Zheng, and C.-H. Chen, "A state-of-the-art survey of Digital Twin: techniques, engineering product lifecycle management and business innovation perspectives," Journal of Intelligent Manufacturing, vol. 31, no. 6, pp. 1313-1337, 2020.

[9] J. Lee, B. Bagheri, and H.-A. Kao, "A Cyber-Physical Systems architecture for Industry 4.0-based manufacturing systems," Manufacturing Letters, vol. 3, pp. 18-23, 2015.

[10] C. Czwick and R. Anderl, "Cyber-physical twins - definition, conception and benefit," Procedia CIRP, vol. 90, pp. 584-588, 2020.

[11] M. Bevilacqua, E. Bottani, F. E. Ciarapica et al., "Digital twin reference model development to prevent operators' risk in process plants," Sustainability, vol. 12, no. 3, pp. 1-17, 2020.

[12] K. Agalianos, S. T. Ponis, E. Aretoulaki, G. Plakas, and O. Efthymiou, "Discrete event simulation and digital twins: review and challenges for logistics," Procedia Manufacturing, vol. 51, pp. 1636-1641, 2020.

[13] A. Saad, S. Faddel, and O. Mohammed, "IoT-based digital twin for energy cyber-physical systems: design and implementation," Energies, vol. 13, no. 18, 2020.

[14] F. Tao, F. Sui, A. Liu et al., "Digital twin-driven product design framework," International Journal of Production Research, vol. 57, no. 12, pp. 3935-3953, 2019.

[15] Q. Yu, J. Ren, Y. Fu, Y. Li, and W. Zhang, "Cybertwin: an origin of next generation network architecture," IEEE Wireless Communications, vol. 26, no. 6, pp. 111-117, 2019.

[16] S. Juneja, A. Juneja, and R. Anand, "Reliability modeling for embedded system environment compared to available software reliability growth models," in Proceedings of the 2019 International Conference On Automation, Computational And Technology Management, London, UK, April 2019.

[17] A. Ahmadi, C. Cherifi, V. Cheutet, Y. Ouzrout, and I. Lyon, "Recent advancements in smart manufacturing technology for modern industrial revolution," A Survey. Joutnal Of Engeneering And Information Science Studies, 2020.
[18] W. Ge and R. Y. Zhong, "Internet of things enabled manufacturing: a review," International Journal of Agile Systems and Management, vol. 11, no. 2, p. 126, 2018.

[19] K. Senthilnathan and I. Annapoorani, "Multi-port current source inverter for smart microgrid applications: a cyber physical paradigm," Electronics, vol. 8, no. 1, pp. 1-20, 2019.

[20] P. K. Padhi and F. Charrua-santos, "6G enabled industrial internet of everything: towards a theoretical framework," Applied System Innovation, vol. 4, no. 1, pp. 1-30, 2021.

[21] A. Fuller, Z. Fan, C. Day, and C. Barlow, "Digital twin: enabling technologies, challenges and open research," IEEE Access, vol. 8, pp. 108952-108971, 2020.

[22] H. Park, A. Easwaran, and S. Andalam, "Challenges in digital twin development for cyber-physical production systems," in Lecture Notes in Computer Science (including subseries Lecture Notes in Artificial Intelligence and Lecture Notes in Bioinformatics)LNCSSpringer International Publishing, New York, NY, USA, 2019.

[23] S. Haag and R. Anderl, "Digital twin-p," Manufacturing Letters, vol. 15, pp. 64-66, 2018.

[24] J. Galvao, J. Machado, G. Prisacaru, D. Olaru, and C. Bujoreanu, "Modelling cyber-physical systems: some issues and directions," IOP Conference Series: Materials Science and Engineering, vol. 444, no. 4, 2018.

[25] I. O. Olalere and O. A. Olanrewaju, "Optimising production through intelligent manufacturing," E3S Web of Conferences, vol. 152, pp. 3-6, 2020.

[26] Y. Natarajan, K. Srihari, G. Dhiman et al., "An IoT and machine learning-based routing protocol for reconfigurable engineering application," IET Communications, pp. 1-12, 2021.

[27] H. K. Upadhyay, S. Juneja, S. Maggu, G. Dhingra, and A. Juneja, "Multi-criteria analysis of social isolation barriers amid COVID-19 using fuzzy AHP," World Journal of Engineering, 2021.

[28] A. Juneja, S. Juneja, A. Soneja, and S. Jain, "Real time object detection using CNN based single shot detector model," Journal of Information Technology Management, vol. 13, no. 1, pp. 62-80, 2021. 